\title{
Three-dimensional optical method for integrated visualization of mouse islet microstructure and vascular network with subcellular-level resolution
}

\author{
Ya-Yuan Fu \\ Chih-Hsuan Lu \\ National Tsing Hua University \\ Department of Chemical Engineering \\ Hsinchu, Taiwan 30013
}

Chi-Wen Lin

National Tsing Hua University

Institute of Biotechnology

Hsinchu, Taiwan 30013

Jyuhn-Huarng Juang

Chang Gung University and Memorial Hospital

Department of Internal Medicine

Division of Endocrinology and Metabolism

Taoyuan, Taiwan 33302

\section{Grigori Enikolopov}

Cold Spring Harbor Laboratory

Cold Spring Harbor, New York 11724

\section{Eric Sibley}

Stanford University

School of Medicine

Division of Pediatric Gastroenterology

Stanford, California 94305

\section{Ann-Shyn Chiang}

National Tsing Hua University

Institute of Biotechnology

Hsinchu, Taiwan 30013

\section{Shiue-Cheng Tang}

National Tsing Hua University

Department of Chemical Engineering

Hsinchu, Taiwan 30013

\section{Introduction}

The islets of Langerhans are small endocrine organs scattered in the pancreas that are crucial for maintaining the glucose homeostasis. The spherical- or ovoid-shaped islet consists of four major secretory cells of insulin, glucagon, somatostatin, and pancreatic polypeptide, i.e., $\beta, \alpha, \delta$, and $\mathrm{F}$ cells, respectively. The balance of the four cell types and the release of the metabolic peptides are tightly regulated by a committee of paracrine, endocrine, and neuronal cues, which poses a chal-

Address all correspondence to Shiue-Cheng Tang, National Tsing Hua University, Department of Chemical Engineering, Hsinchu, Taiwan 30013. Tel: 886-3 571-5131, ext. 33649. Fax: 886-3-571-5408; E-mail: sctang@che.nthu.edu.tw

\begin{abstract}
Microscopic visualization of islets of Langerhans under normal and diabetic conditions is essential for understanding the pathophysiology of the disease. The intrinsic opacity of pancreata, however, limits optical accessibility for high-resolution light microscopy of islets in situ. Because the standard microtome-based, 2-D tissue analysis confines visualization of the islet architecture at a specific cut plane, 3 -D representation of image data is preferable for islet assessment. We applied optical clearing to minimize the random light scattering in the mouse pancreatic tissue. The optical-cleared pancreas allowed penetrative, 3-D microscopic imaging of the islet microstructure and vasculature. Specifically, the islet vasculature was revealed by vessel painting - lipophilic dye labeling of blood vessels_-for confocal microscopy. The voxel-based confocal micrographs were digitally processed with projection algorithms for 3-D visualization. Unlike the microtome-based tissue imaging, this optical method for penetrative imaging of mouse islets yielded clear, continuous optical sections for an integrated visualization of the islet microstructure and vasculature with subcellular-level resolution. We thus provide a useful imaging approach to change our conventional planar view of the islet structure into a 3-D panorama for better understanding of the islet physiology. () 2010 Society of Photo-Optical Instrumentation Engineers. [DOI: $10.1117 / 1.3470241]$
\end{abstract}

Keywords: 3-D microscopy; islets of Langerhans; microstructure; vasculature.

Paper 10110R received Mar. 6, 2010; revised manuscript received Jun. 2, 2010; accepted for publication Jun. 3, 2010; published online Aug. 4, 2010. lenging task of analyzing the islet physiology as well as its critical disorder-diabetes mellitus. ${ }^{1,2}$

Anatomically, the endocrine islets are surrounded by the exocrine acini and constantly receive paracrine signals from the neighboring acinar cells. In addition, islets are densely vascularized, receiving 5 to $15 \%$ of the pancreatic blood supply, despite contributing only approximately $1 \%$ to the pancreatic volume. ${ }^{3,4}$ The structural and functional connections between the islet endocrine cells and vascular endothelial cells are important to provide oxygen and nutrients to the endocrine cells and allow rapid transport of endocrine hor-

1083-3668/2010/15(4)/046018/9/\$25.00 @ 2010 SPIE 

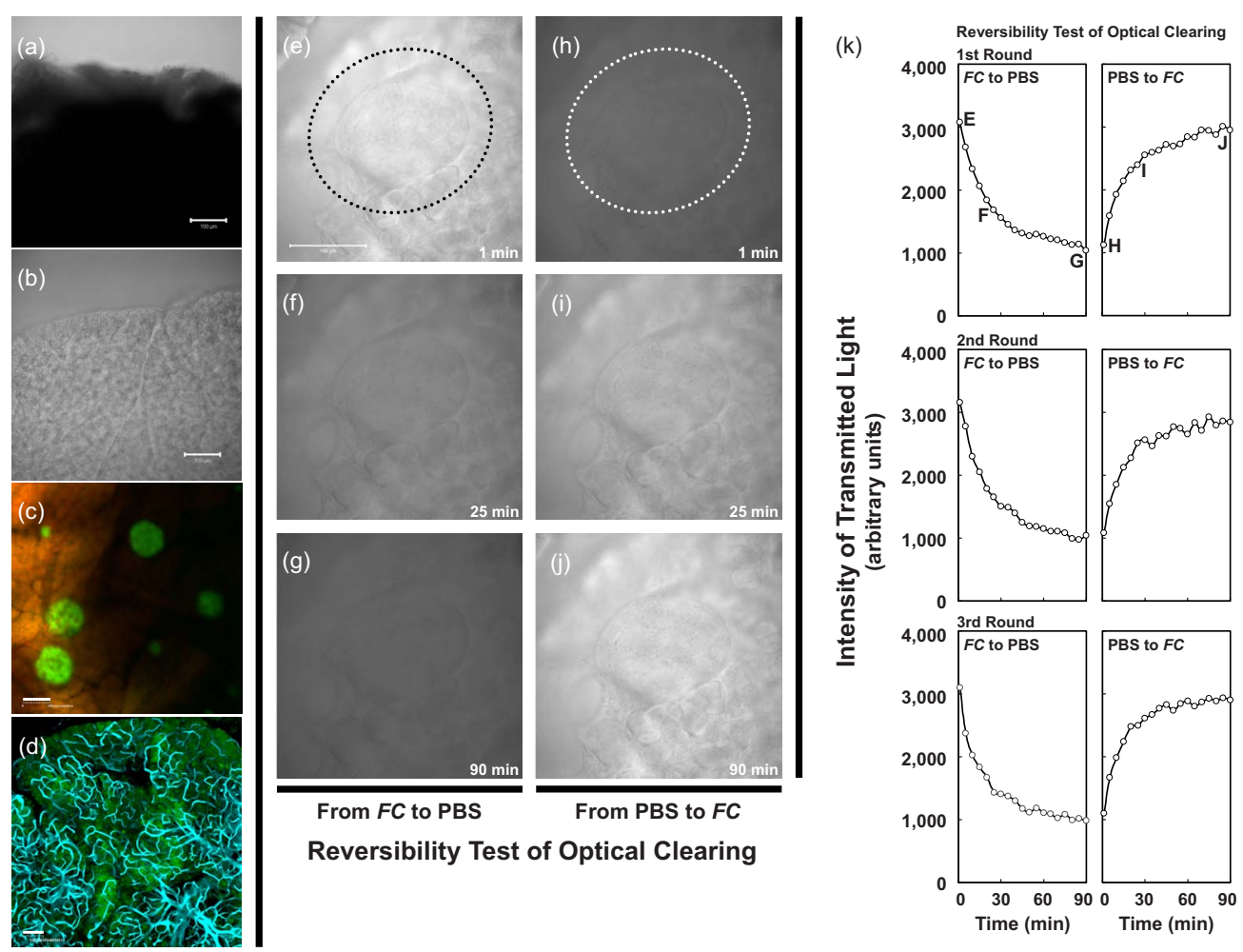

Fig. 1 (a) and (b) Optical clearing improves the transparency of the mouse pancreas. The specimens were immersed in phosphate-buffered saline (PBS) (a) or FocusClear solution (b) prior to being imaged by the transmitted light channel of confocal microscopy. (c) and (d) Gross images of islets of Langerhans (revealed by insulin immunostaining) and vasculature (revealed by vessel painting), respectively, in the optical-cleared pancreas. (e) to (j) Reversibility test of the optical clearing effect. The intensity of transmitted light is presented in gray scale. In (e) to (g) and (h) to (j), the immersion solution was changed from FocusClear to PBS and from PBS to FocusClear, respectively. FC: FocusClear. The dotted circle indicates the location of the islet. (k) Kinetics of the optical clearing effect and the reverse of optical clearing. The intensity of transmitted light was measured over time. A similar trend was seen in all three rounds of optical clearing. The letters e to $\mathrm{j}$ in the upper panel of $(\mathrm{k})$ correspond to the grayscale micrographs of (e) to (j). The volume ratio of tissue/FocusClear is $\sim 1 / 50$. In (a) to (e), bar $=100 \mu \mathrm{m}$.

mones such as insulin to the circulation for regulation of blood glucose levels.

Although there is an intensive need to characterize the islet microstructure and vasculature in an integrated fashion, visualization of the pancreatic islet and its vascular network is limited by the spatial resolution of the imaging tools. In routine laboratory practice, the microtome-based imaging method allows $\mu \mathrm{m}$-level resolution but confines the view at a specific cut plane. In addition, the disconnection between sectioned tissues as well as the distortions and artifacts caused by microtome slicing prevent an integral, 3-D visualization of the islet in situ.

For transparent tissues such as the retina, where light can pass through with minimal scattering, optical imaging such as confocal microscopy provides a useful tool to study the 3-D tissue structure with appropriate fluorescent labeling. Unfortunately, pancreatic tissues are nontransparent [Fig. 1(a)], which seriously limits their optical accessibility. Recent progress in optical clearing of tissues and cells, ${ }^{5-11}$ however, sheds light on deep-tissue microscopy by improving the tissue optics for imaging. Tissues such as insect brains, ${ }^{12-14}$ mouse intestines, ${ }^{15}$ and tissue-engineering constructs ${ }^{16}$ were immersed in optical-clearing solution to reduce refractive mismatch between the tissue constitutes (refractive index at 1.46) and fluids (water has a refractive index at 1.33). This avoids random scattering when light moves between media, therefore facilitating laser penetration and fluorescence detection in confocal microscopy. ${ }^{17}$ In this research, we integrated optical clearing with confocal microscopy to achieve in situ, 3-D imaging of mouse pancreatic islets with subcellular-level resolution. Using this imaging approach, for the first time we were able to apply vessel painting (lipophilic dye labeling of blood vessels) ${ }^{18,19}$ to visualize the islet/pancreatic vasculature network with high definition, which is critically missing in previous studies of optical imaging of islets. ${ }^{20,21}$ Panoramas of the mouse islet and its tissue network and our approach to acquire the 3-D images are presented and discussed.

\section{Materials and Methods}

\subsection{Animals}

Pancreata from the female wild-type BALB/c mice (BioLASCO, Taipei, Taiwan) were used in insulin staining and tests of optical clearing. The tissues were also used in membrane and nuclear staining to examine the islet/pancreatic microstructure. Pancreata from the nestin-green fluorescent protein (GFP) transgenic mice were used in vessel painting to examine the islet microvasculature. In these transgenic animals, the GFP expression is under the control of the $5.8-\mathrm{kb}$ protomer and the $1.8-\mathrm{kb}$ second intron of the nestin gene, which encodes a type VI intermediate filament protein. ${ }^{22} \mathrm{Par}-$ ticularly, in the pancreas, this mouse line shows strong GFP 
expression in the exocrine acinar cells and, minimal, if any, GFP expression in the endocrine islet cells. ${ }^{23}$ Overall, 15 and 7 wild-type BALB/c (36 islets) and nestin-GFP (32 islets) mice were used, respectively, to generate representative images. The care of the animals was consistent with Guidelines for Animal Experiments, National Tsing Hua University, Taiwan.

\subsection{Sample Preparation}

Mouse pancreata were fixed by paraformaldehyde (4\%) perfusion alone (used in BALB/c mice) or vessel painting of lipophilic dye DiD (4-chlorobenzene sulfonate salt, purchased from Invitrogen, Carlsbad, California, used in the nestin-GFP mice $)^{18,19}$ followed by paraformaldehyde perfusion prior to being harvested for examination. Once harvested, the tissues were fixed in paraformaldehyde $(4 \%)$ for an additional $1 \mathrm{~h}$. Mouse insulin staining was achieved by incubating the specimen with the primary rabbit-anti-mouse insulin antibody (1:50, Cell Signaling, Danvers, Massachusetts) and then revealing with Alexa Fluor 488-conjugated goat-anti-rabbit IgG (1:200, Invitrogen). Propidium iodide staining (PI staining, $25 \mu \mathrm{g} / \mathrm{ml}$, Invitrogen) was performed at room temperature for $90 \mathrm{~min}$ to label the nuclei. DiD staining of membranes $(2 \mu \mathrm{g} / \mathrm{ml})$ was performed at room temperature overnight. Specimens from the DiD-perfused nestin-GFP mice (vessel painting) were stained with PI only. The labeled specimens were immersed in FocusClear solution (CelExplorer, Hsinchu, Taiwan) for optical clearing before being imaged via confocal microscopy.

\subsection{Imaging Settings}

Confocal imaging of the optical-cleared specimens was performed with a Zeiss LSM 510 confocal microscope equipped with a $25 \times$ objective LD Plan-Apochromat glycerine immersion lens or $40 \times$ objective LD C-Apochromat water immersion lens. A $10 \times$ objective Fluar was used to acquire gross images. The PI- and DiD- labeled samples were excited with helium/neon lasers at $543 \mathrm{~nm}$ and $633 \mathrm{~nm}$, respectively. A bandpass 560- to 615-nm filter and a long-pass 650-nm filter were used to collect signals from PI and DiD, respectively. A 488-nm argon laser and a bandpass 505- to 530-nm filter were used for GFP excitation and fluorescence detection. In addition to fluorescence imaging, the Zeiss LSM 510 confocal microscope carries the function of transmission light imaging, which can be integrated with the confocal imaging when the detector of the transmitted light channel $(\mathrm{ChD})$ is open. The imaging process is similar to viewing a thick, transparent tissue using a standard microscope with an additional function so that the focal path can be digitally adjusted in coordination with the confocal imaging. That is, while the detector of the confocal channel is collecting the signals from a single plane, the detector of the transmitted light channel is collecting the laser light traveling across the tissue.

Sample scanning was recorded with $1024 \times 1024$ pixels of the $x / y$ plane. The increment of the $z$-axis optical section was $2.5 \mu \mathrm{m}$ when $10 \times$ objective lenses were used to acquire gross images. The increment was 1 or $1.5 \mu \mathrm{m}$ when $25 \times$ and $40 \times$ objective lenses were used to acquire high-resolution images. The pixel intensity was set to be the mean of four

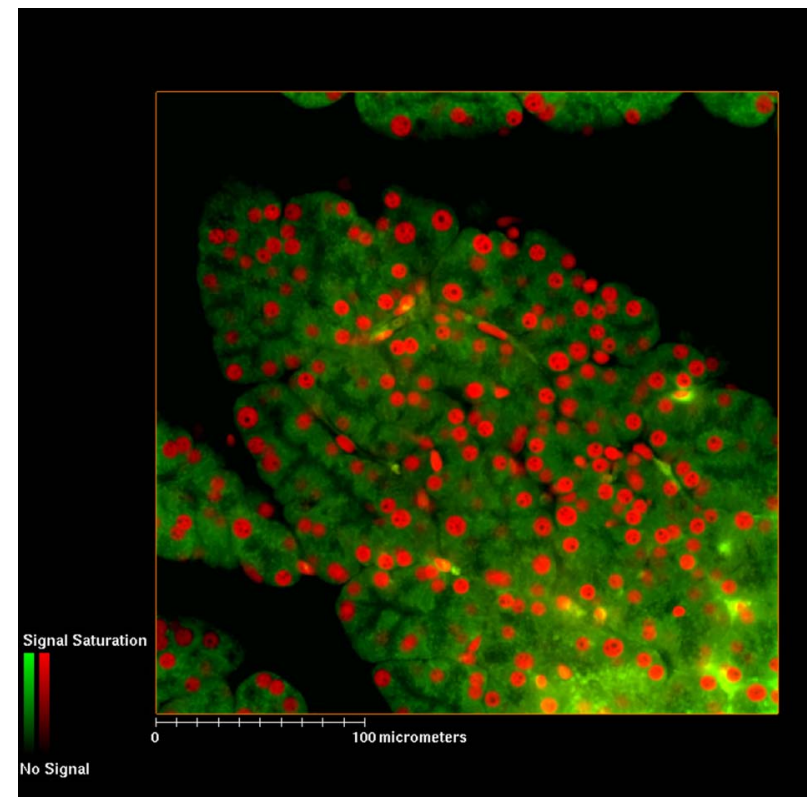

Video 1 Complete serial optical sections of the mouse pancreas shown in Figs. 2(a)-2(e) (depth $=330 \mu \mathrm{m})$. The specimen was stained by $\mathrm{DiD}$ and propidium iodide to reveal the cellular membranes (green) and nuclei (red), respectively. Two islets were imaged in the scanned volume at depths of $\sim 180 \mu \mathrm{m}$ and $\sim 270 \mu \mathrm{m}$ (MPEG 4.9 MB). (Color online only.)

[URL: http://dx.doi.org/10.1117/1.3470241.1].

scans, and the value ranged from 0 to 4095 (12-bit data) for the transmitted light imaging and from 0 to 255 (8-bit data) for the rest of the confocal imaging.

\subsection{Post-Recording Image Processing and Projection}

The voxel-based confocal micrographs were processed with the use of the LSM 510 software (Version 3.2, Carl Zeiss) and the Amira 4.1.2 (Mercury, Chelmsford, Massachusetts) for projection and analysis. Typically, for a three-channel confocal microscopy (such as to collect signals from GFP, PI, and DiD), the size of the image data was $\sim 630$ megabytes $(1024 \times 1024$ pixels $\times 200$ slices $\times 3$ channels of signals $)$ for an imaging depth of $300 \mu \mathrm{m}$. The LSM image data were uploaded into Amira, which was operated under a Dell Precision T5400 workstation. We applied the Gaussian Filter function of Amira for noise reduction of the micrographs. In Videos 1, 2, and 5 the image stacks were displayed using the Ortho Slice function, and the video was made via the Movie Maker function with an increase in display time in association with the depth of the optical section. In Videos 2 to 4, 6, and 7, the Voltex module of Amira was used to project the image stacks. In addition, the Volume Editing function was used to subtract a cuboid(s) from the scanned volume to expose the interior domain of the specimen for visualization. In video preparation, the Demo Maker function was used to arrange the sequence of the image objects at different time intervals. The Camera Path function was used to adjust the projection angles and zoom-in and zoom-out movements of the 3-D images. 


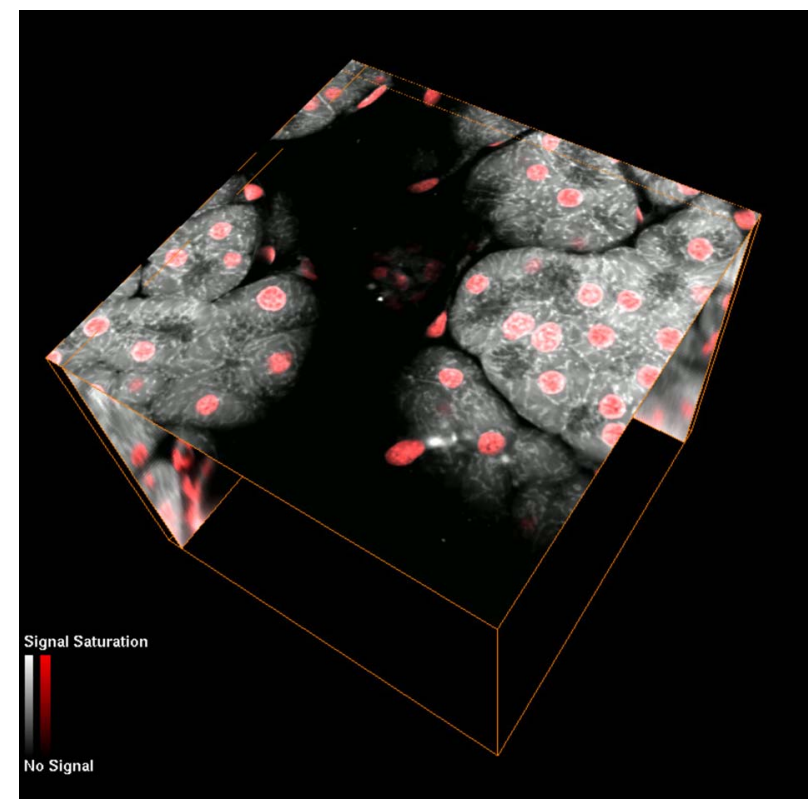

Video 2. High-resolution, stereo projection of the serial orthogonal views of the islet shown in Figs. 2(i)-2(k). The specimen was stained by DiD and propidium iodide to reveal the cellular membranes (gray) and nuclei (red), respectively. Dimensions of the scanned volume: $175 \mu \mathrm{m}(x) \times 175 \mu \mathrm{m}(y) \times 122 \mu \mathrm{m}$ ( $z$, depth) (MPEG 4.9 MB). (Color online only.) [URL: http://dx.doi.org/10.1117/1.3470241.2].

\section{Results}

\subsection{Optical Clearing of the Mouse Pancreas}

We applied an optical-clearing solution FocusClear (U.S. Patent No. 6472216) ${ }^{24}$ to facilitate photon penetration across the mouse pancreatic tissue. The optical-clearing effect allowed the opaque specimen [Fig. 1(a), in saline] to become transparent when it was immersed in the FocusClear solution [Fig. 1(b)]. In the transparent pancreas, we were able to visualize islets [Figure 1(c), insulin immunostaining] and the vascular structure [Figure 1(d), details described later] in situ. Traditionally, pancreata are sectioned into thin slices (e.g., $10 \mu \mathrm{m}$ in thickness) to expose their interior domain for microscopic examination. ${ }^{1}$ Here, the image area can be as large as $1000 \mu \mathrm{m}(x) \times 1000 \mu \mathrm{m}(y) \times 400 \mu \mathrm{m}(z$, depth). The thickness of the specimen can be in the $800-\mu \mathrm{m}$ range, given that separate visuals can be made from both sides of the specimen.

A significant feature of the FocusClear-mediated optical clearing is its reversibility. Figure 1(e) shows an islet residing in the transparent pancreas, which was acquired using the transmitted light channel of confocal microscopy. When we replaced FocusClear with saline [Figs. 1(e)-1(g)] and then repeated the optical-clearing immersion [Figs. 1(h)-1(j)], the optical-clearing effect was reproduced [Figs. 1(e)-1(j)]. We measured the kinetics of this effect: Figure 1(k) shows a similar trend in all three tests, confirming the reversibility of the FocusClear-mediated optical clearing. Note that in sample preparation, paraformaldehyde was used to fix the specimen prior to the FocusClear immersion. Importantly, the size of the islet in the paraformaldehyde-fixed specimen remained the same, while the tissue optics changed during the test [Figs. $1(\mathrm{e})-1(\mathrm{j})$ _ - this is essential with the use of a new optical

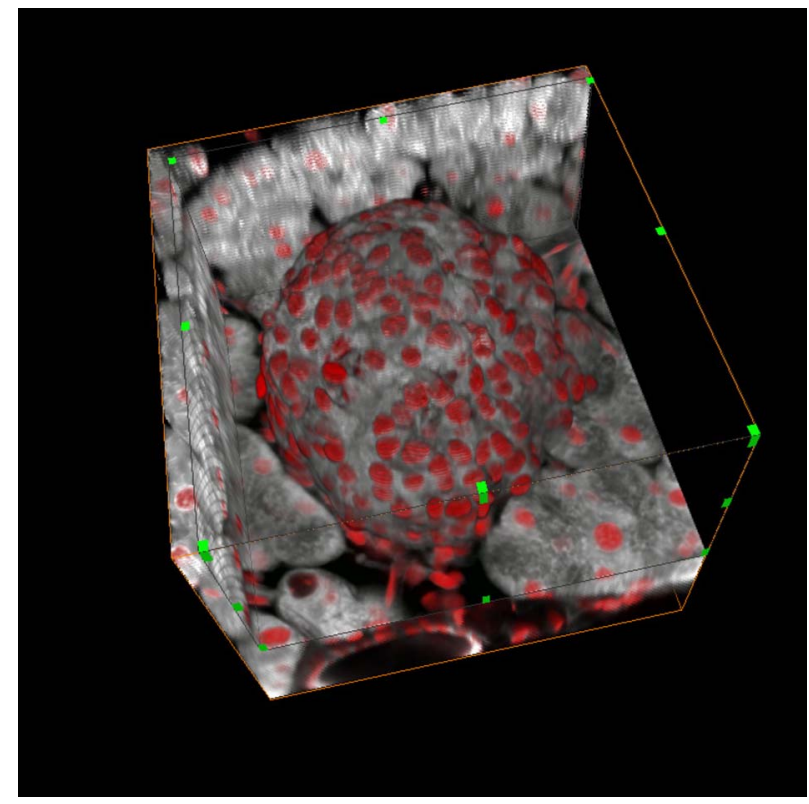

Video 3. 360-deg panoramic presentation of Fig. 2(k). The voxels of the islet shown in Video 2 were digitally collected and merged with the signals shown in Fig. 2(j) (MPEG 5 MB).

[URL: http://dx.doi.org/10.1117/1.3470241.3].

method for tissue examination. However, we would like to stress the finding by Goldstein et al. that upon comparison of the freshly excised and the formalin-treated specimens, there was shrinkage after fixation. ${ }^{25}$ Thus, there are still disparate observations in the size of tissue structures between in vivo and in vitro measurements.

\subsection{Penetrative Confocal Imaging of Islets of Langerhans in the Mouse Pancreas}

Taking advantage of the transparent specimen, we next applied deep-tissue confocal microscopy to examine the fluorescent-dye-labeled structure. Figures 2(a)-2(e) are examples of the confocal micrographs at different depths in the specimen, where the nuclei and membranes were revealed by propidium iodide (PI) and DiD (4-chlorobenzene sulfonate salt) staining, respectively. The complete serial optical sections, from the surface to depth $=330 \mu \mathrm{m}$, are shown in Video 1, which reveals two islets in the imaged region. Because the two islets are embedded in the exocrine acini, we used the Amira image software to digitally subtract two cuboids from the scanned volume to expose their locations [Figs. 2(f)-2(h)].

In a second example, Figs. 2(i)-2(k), we used a higher magnification to examine the pancreatic islet structure. Video 2 uses stereo projection to display continuous orthogonal views of the imaged islet. Because there were no physical sections involved in sample preparation, we can digitally quantify the size of the islet by gathering the islet signals based on their locations in the micrograph and calculating the occupied voxels. For example, the size of the scanned volume shown in Figs. $2(\mathrm{i})-2(\mathrm{k})$ is $175 \times 175 \times 122 \mu \mathrm{m}^{3}$, and the size of each voxel is $0.17 \times 0.17 \times 1.5 \mu \mathrm{m}^{3}$. By calculating the voxels occupied by the islet [Fig. $2(\mathrm{k})$ ], we estimate that the volume of the islet is $7.1 \times 10^{5} \mu \mathrm{m}^{3}$ in the specimen. A 


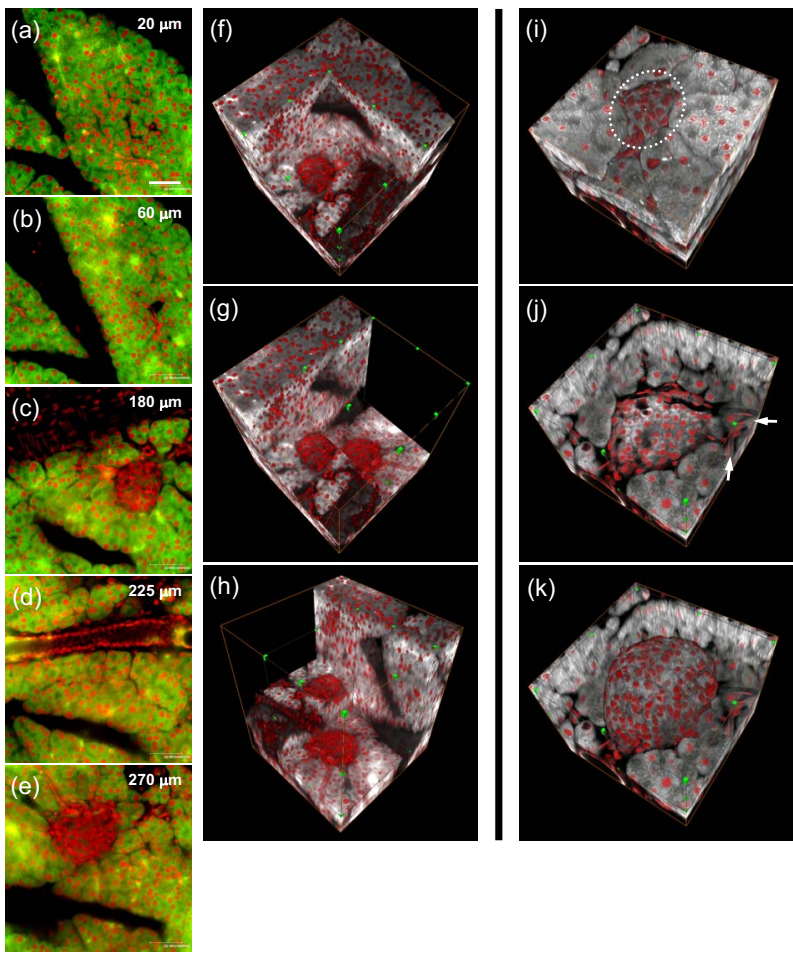

Fig. 2 Penetrative confocal imaging of islets of Langerhans in the mouse pancreas. (a) to (e) Confocal micrographs at different depths in the pancreatic tissue specimen. (a) and (b) The exocrine acinar tissue. (c) to (e) Islets of Langerhans in association with acinar cells, pancreatic duct (d), and blood vessels. Cells in the specimen were stained by $\mathrm{DiD}$ and PI to reveal the cellular membranes (green) and nuclei (red), respectively. Particularly in (e), the elongation of the endothelial cell nuclei along the vessel direction can be clearly identified. Bar $=50 \mu \mathrm{m}$. The complete serial optical sections, from the surface to depth $=330 \mu \mathrm{m}$, are shown in Video 1. (f) and (g) Stereo projections of the stack of confocal micrographs showing the two islets [(c) and (e)] embedded in the exocrine acini and linked with blood vessels. The DiD-stained cellular membranes are presented in gray scale for 3-D perspective views. In (f), a cuboid was digitally subtracted from the scanned volume to reveal the islet shown in (c). In (g), a second cuboid was subtracted from ( $f$ ) to present both of the two islets shown in (c) and (e). (h) A clockwise, 90-deg rotation of (g) was made to create a new viewing angle. Dimensions of the scanned volume: $290 \mu \mathrm{m}(x) \times 290 \mu \mathrm{m}(y) \times 330 \mu \mathrm{m} \quad(z$, depth). (i) to (k) Highresolution, 3-D projections of an embedded islet in connection with its vascular network. In (i), the dotted circle indicates the location of the embedded islet. In (j), a cuboid was subtracted to expose the capillary (arrows). In (k), the fluorescent signals of the islet were digitally extracted from the original stack of confocal micrographs and then added into (j). Continuous orthogonal views of the islet and a 360-deg panoramic presentation of $(\mathrm{k})$ are shown in Videos 2 and 3, respectively. (Color online only.)

360-deg panoramic presentation of Fig. 2(k) is shown in Video 3. This approach allows an integral view and assessment of the islet structure, which cannot be done by the standard microtome-based 2-D analysis.

\subsection{High-Resolution, 3-D Imaging of Islet Vasculature in the Mouse Pancreas}

Vessel painting is a direct staining method that uses perfusion of lipophilic dye to label blood vessels in small experimental animals. Previously, this method has been used to visualize

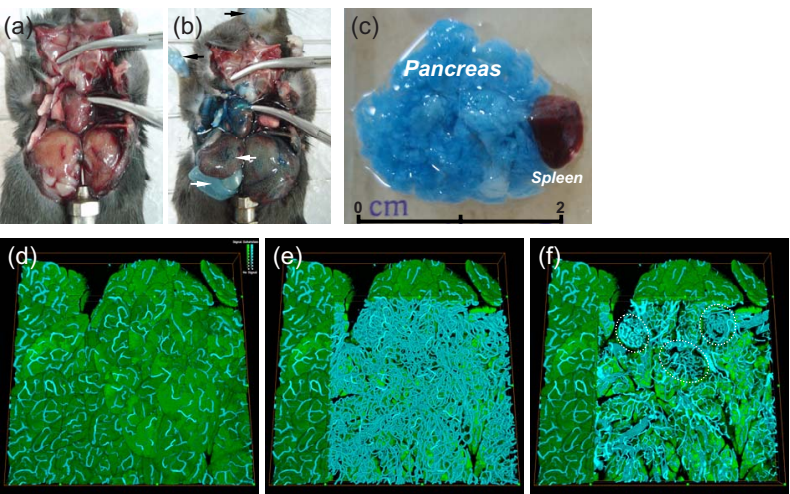

Fig. 3 (a) to (c) Use of vessel painting, i.e., lipophilic dye DiD perfusion, to label blood vessels. (a) Prior to vessel painting and (b) post vessel painting of the nestin-GFP transgenic mouse. Arrows in (b) indicate the stained blue tissues/organs after the DiD perfusion. (c) An enlarged view of the harvested mouse pancreas (together with the spleen) after vessel painting. (d) to (f) 3-D gross views of the labeled pancreatic tissue. Signals of GFP expression (green) and DiD-labeled vasculature (cyan) are combined in (d). In (e), a cuboid (depth $=125 \mu \mathrm{m}$ ) of GFP signals is subtracted from (d) to reveal the vasculature. In (f), a cuboid (depth $=80 \mu \mathrm{m}$ ) of DiD signals is subtracted from (e) to reveal the locations of the three embedded islets, indicated by the white dotted circles. Dimensions of the scanned volume: $1300 \mu \mathrm{m}(x) \times 1300 \mu \mathrm{m}(y) \times 250 \mu \mathrm{m}(z$, depth). Video 4 provides additional projection angles and zoom-in and zoom-out movements of panels (d) to (f). In the 3-D projections, we added an $x / y$ plane at $z=125 \mu \mathrm{m}$ to outline the tissue structure. (Color online only.)

the vasculature of the retina, skin, lung, heart, and brain in mice, but with limited imaging depth. ${ }^{18,19}$ Here, we tested the compatibility of vessel painting with our optical method to visualize the mouse islet/pancreatic vasculature in a 3-D fashion. Figures 3(a)-3(c) show that the mouse tissues, including the pancreas, turned blue after perfusion with the lipophilic dye DiD. The labeled pancreatic tissue was then optically cleared by the FocusClear solution and underwent confocal microscopy for deep-tissue imaging. This approach led to a clear visualization of the vascular network, including the capillaries in the islet. Figures 3(d)-3(f) are the gross views of the labeled pancreatic tissue, where there are three embedded islets, residing at 50 to $200 \mu \mathrm{m}$ under the tissue surface. In Fig. 3(e), we used the Amira software to digitally subtract a cuboid of signals from acini to reveal the vasculature inside. In Fig. 3(f), we subtracted the vasculature signals on top of the cuboid (from 0 to $80 \mu \mathrm{m}$ in depth) to reveal the locations of the three islets. Stereo projections of Figs. 3(d)-3(f) are shown in Video 4.

We next zoomed in to visualize the islet vasculature with high resolution. The optical sections at different depths of a typical islet are shown in Figs. 4(a)-4(e). Images from the transmitted light and fluorescence channels of confocal microscopy are presented on the left and right sides of the figure, respectively. Video 5 displays the serial optical sections of the scanned region. Specifically, on the left side of Fig. 4(e), large blood vessels can be seen using transmitted light imaging. On the right side, these vessels are also revealed by fluorescence imaging, confirming the success of vessel painting. Notably, the microvessels with a diameter of $\sim 5 \mu \mathrm{m}$ are clearly visualized in the islet and acini. 

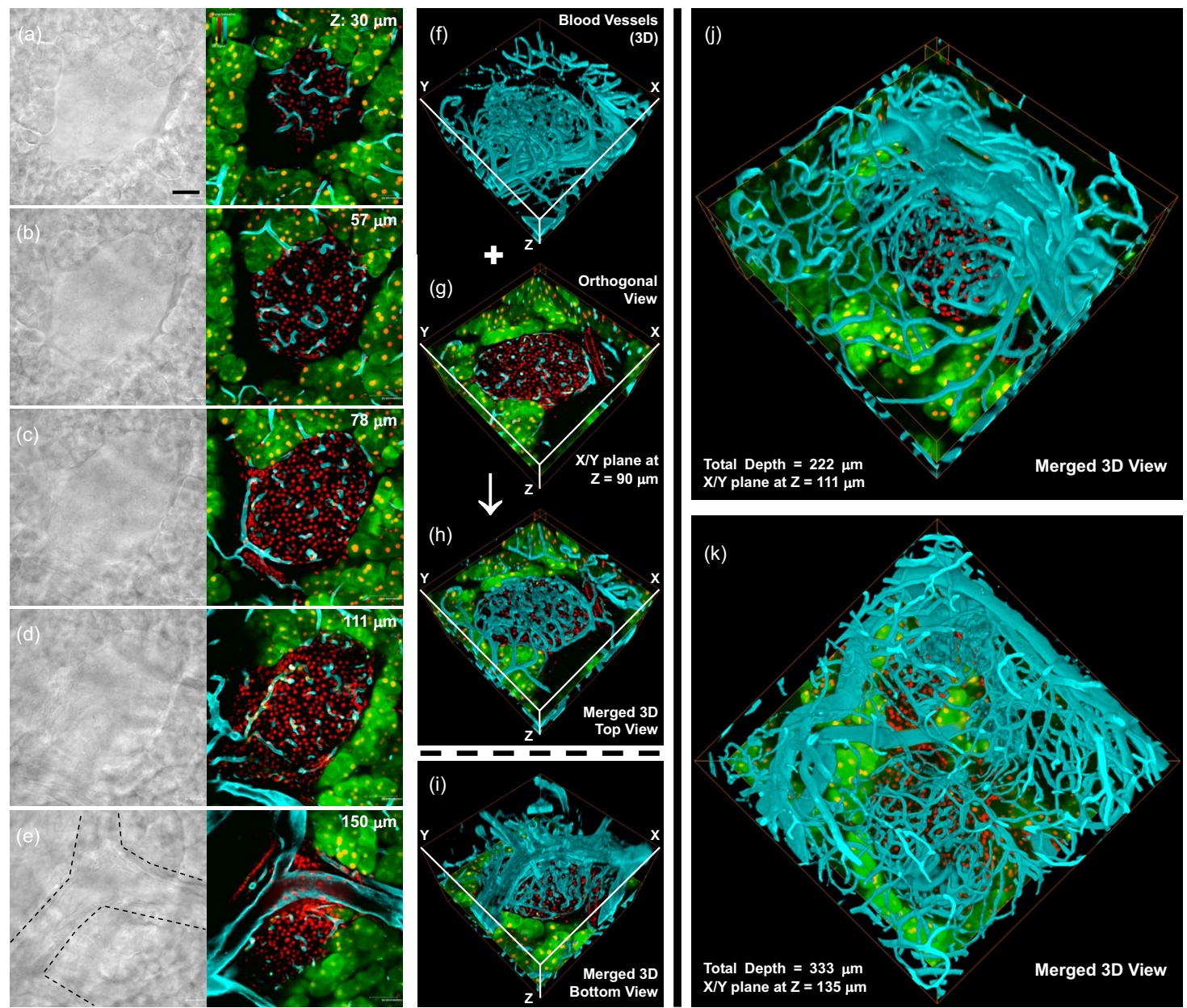

Fig. 4 High-resolution, 3-D images of the islet vasculature in the mouse pancreas. (a) to (e) Micrographs of transmitted light (left) and fluorescence (right) imaging at different depths in the pancreatic tissue specimen. The vascular network (cyan) was labeled with lipophilic DiD by cardiac perfusion. PI staining was used to reveal the nuclei (red). We used the pancreas from the nestin-GFP transgenic mice (GFP expression driven by the nestin promoter) to highlight the exocrine acini (green) (Refs. 21 and 22) This mouse line shows minimal, if any, GFP expression in the islet endocrine cells. In (e), large blood vessels can be seen in both the transmitted light (dotted lines indicate the approximate boundaries) and the fluorescence micrographs. The complete fluorescent serial optical sections of the imaged islet are shown in Video 5. (f) to (i) Separate and merged stereo projections of the blood vessels and the islet/pancreatic structure. (h) and (i) are projections from the top and bottom halves of the imaged region. A "landmark" pancreatic duct can be seen in (g), at the right side of the islet. The same duct can be seen in (h) and (i), yet some areas were blocked by the projection of blood vessels. Dimensions of the scanned volume: $369 \mu \mathrm{m}(x) \times 369 \mu \mathrm{m}(y) \times 225 \mu \mathrm{m}(z$, depth). (j) and (k) Representative 3-D projections of the islet vasculature in the mouse pancreas. Video 6 provides additional projection angles and zoom-in and zoom-out movements of panel (k). (Color online only.)

Figures $4(\mathrm{f})$ and $4(\mathrm{~g})$ are stereo projections of the blood vessels and an orthogonal view of the islet shown in Figs. 4(a)-4(e). The 3-D top view and bottom view of the islet vasculature, shown in Figs. 4(h) and 4(i), are created by using the Amira software to merge the signals shown in Figs. 4(f) and $4(\mathrm{~g})$ and rotate the viewing angle to project the top and bottom halves of the imaged region. Figures $4(\mathrm{j})$ and $4(\mathrm{k})$ are two additional results of the high-resolution, 3-D vasculature imaging of the mouse pancreatic islets. A fly-through presentation of Fig. 4(k) is shown in Video 6. Furthermore, in Video 7 , we applied the Image Segmentation function of the Amira software to highlight the vasculature in the interior and exterior domains of the islet. These results explore the feasibility of an in situ 3-D image comparison between the endocrine and exocrine parts of the pancreas, which could be used to examine the remodeling of microvessels during the progression of autoimmune diabetes.

\section{Discussion}

The worldwide prevalence of diabetes mellitus underlines the complexity of the disease and an urgent need to better understand the islet physiology and developing diagnostic and monitoring tools for the disease. In this research, we develop a new imaging approach to extend our conventional planar view of the microscopic islet/pancreatic architecture into a 3-D panorama for characterization of islets in situ. In our approach, we explored the efficiency of an optical-clearing reagent, FocusClear, in improving photon penetration in the mouse pancreatic tissue. We engineered the tissue optics to 


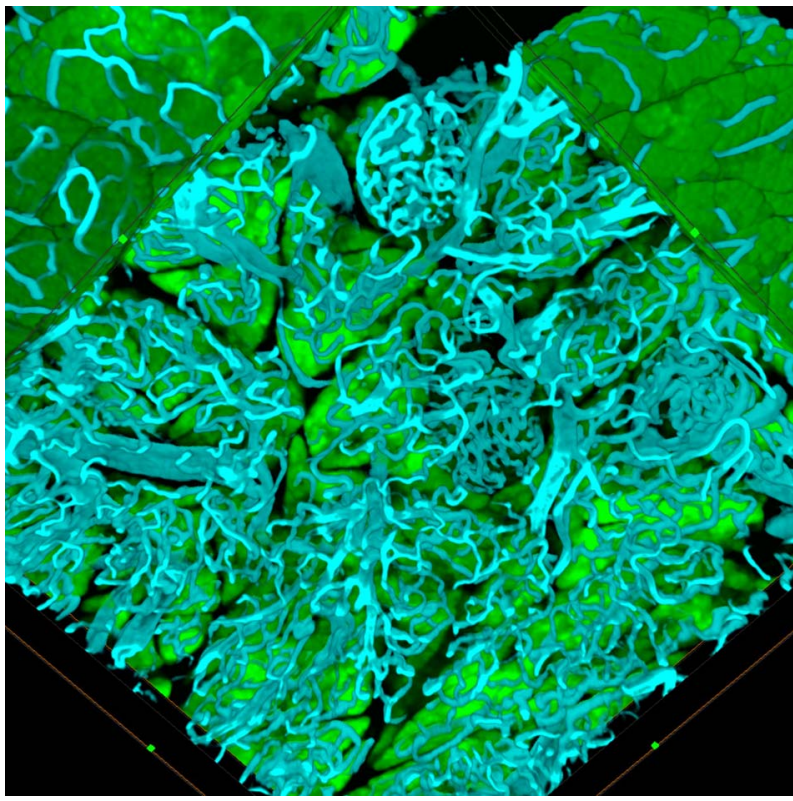

Video 4. 3-D gross views of the mouse pancreatic vasculature. Lipophilic dye DiD perfusion was used to label blood vessels. The Volume Editing function of the Amira software was used to subtract signals from acini (green) and then vasculature (cyan) at the top corner of the imaged region to expose the interior domain for visualization. Dimensions of the scanned volume: $1300 \mu \mathrm{m}(x) \times 1300 \mu \mathrm{m}(y) \times 250 \mu \mathrm{m}$ ( $z$, depth). An $x / y$ plane is shown at $z=125 \mu \mathrm{m}$ to outline the tissue structure (MPEG 4.8 MB). (Color online only.)

[URL: http://dx.doi.org/10.1117/1.3470241.4].

reveal the embedded islets in the pancreas, where random light scattering was suppressed and the size of the islet in the paraformaldehyde-fixed specimen remained the same. Using fluorescent labeling, the islet/pancreatic microstructure and vasculature were revealed by $3-\mathrm{D}$ confocal microscopy with efficient fluorescence excitation and emission in the opticalcleared specimen. The 3-D figures and videos presented in this report make possible viewing the spatial relationship of the endocrine islet and the exocrine acini. Significantly, because microtome sectioning is not involved in sample preparation, our approach provides a useful tool for an integral, 3-D presentation of the intricate islet microvasculature.

Recently, significant progress has been made in developing optical methods for in vivo imaging of islet cell mass and vasculature. ${ }^{26-29}$ For example, Nyman et al. ${ }^{28}$ used confocal microscopy to examine the blood flow in the exteriorized pancreatic islets. Speier et al. ${ }^{29}$ transplanted islets into the anterior chamber of the mouse eye for a transparent environment to study islet cell biology via two-photon microscopy. However, the extension of confocal and two-photon microscopy to in vivo imaging still leaves unresolved the problem of light scattering as photons encounter the opaque tissue. In two-photon microscopy, for instance, although the infrared lasers excite fluorophores twice as deep in comparison to confocal microscopy, the resolution is not significantly improved because the emitted fluorescence still needs to travel through the turbid tissue for detection. Evidently, in both Nyman's and Speier's studies, images were limited at cellular-level resolution, in addition to lacking a clear view of the vascular structure in the interior domain of the islet. Results of these studies confirm

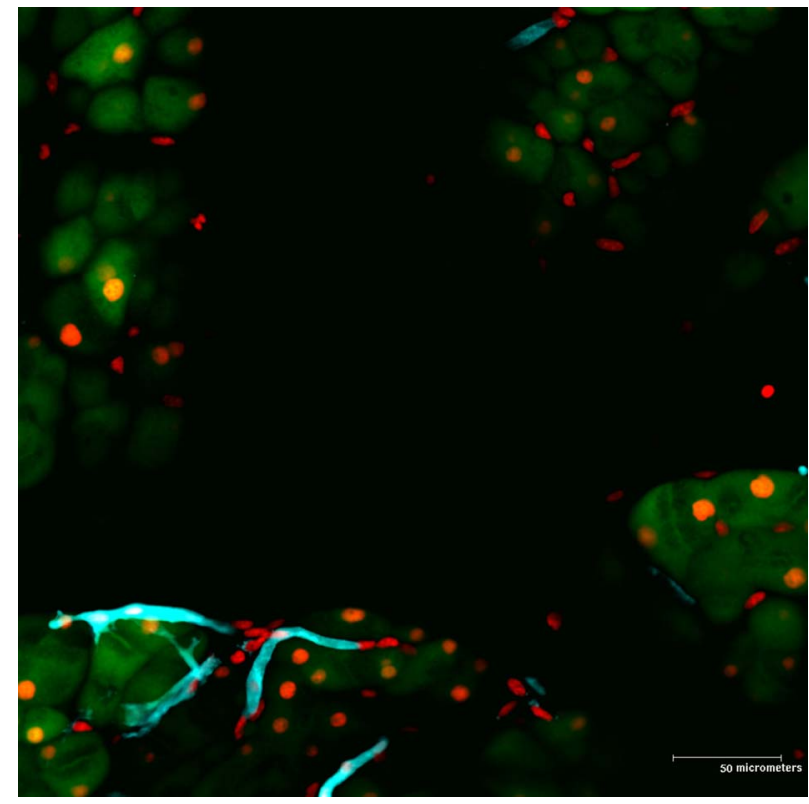

Video 5. High-resolution, serial optical sections of the islet vasculature shown in Figs. 4(a)-4(e) (depth=225 $\mu \mathrm{m})$. Fluorescent labeling of blood vessels (cyan) was done by cardiac perfusion of DiD, i.e., vessel painting. The pancreas from the nestin-GFP transgenic mouse was used in the imaging, which has strong nestin-GFP expression in the exocrine acinar cells (green). Propidium iodide staining was used to reveal the nuclei (red) (MPEG $5 \mathrm{MB}$ ). (Color online only.) [URL: http://dx.doi.org/10.1117/1.3470241.5].

that the process of optical clearing is essential in using optical microscopy to acquire high-definition images of the microvascular network, as shown in Figs. 3 and 4. However, it should be noted that the optical-clearing-enhanced microscopy can be done only on a deceased animal, so the ability to perform longitudinal measurements is restricted to a single time point. Although in vivo microscopy is limited due to optical scattering, a key advantage remains that it can be done in a longitudinal fashion. Therefore, both approaches carry pros and cons.

In this study, we applied the FocusClear solution (U.S. Patent No. 6472216) ${ }^{24}$ for optical clearing to improve photon penetration in the pancreatic tissue. FocusClear is an aqueous mixture of chemicals consisting of sugars and their derivatives with high refractive indices $(\sim 1.46)$ similar to those of the tissue constitutes. Immersion of pancreatic tissues in the solution reduces the amount of refractive mismatch between tissue constitutes and the surrounding fluids, thereby avoiding scattering and making the pancreatic tissue transparent for optical imaging.

In addition to optical microscopy, advances have been made in using noninvasive imaging methods, such as magnetic resonance imaging (MRI) and positron emission tomography (PET), in animal models to detect the altered $\beta$-cell mass and the microvascular remodeling during the progression of type 1 (autoimmune) diabetes. ${ }^{26,27,30-36}$ MRI, for example, can image deep into the pancreas in vivo and provide 3 -D information such as the angiogram. ${ }^{30-32}$ Continued advances in noninvasive monitoring of the pathophysiological islet changes would provide crucial help in early diagnosis and therapeutic intervention of the disease. However, to date, the spatial resolution of MRI and PET is too low to observe 


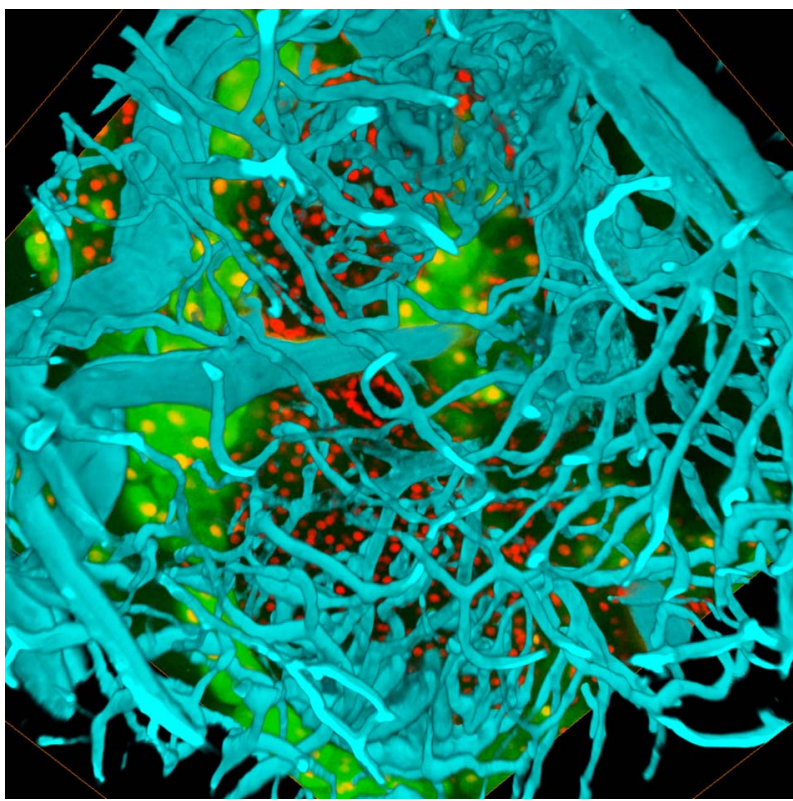

Video 6. Fly-through presentation of the islet vasculature shown in Fig. 4(k) using multiple projection angles and magnifications. The Camera Path function of the Amira software was used to adjust the viewing angles and the zoom-in and zoom-out movements. Dimensions of the scanned volume: $521 \mu \mathrm{m}(x) \times 521 \mu \mathrm{m}(y) \times 333 \mu \mathrm{m}(z$, depth). Two $x / y$ planes are shown at $Z=135$ and $204 \mu \mathrm{m}$ to outline the tissue structure. Two islets reside in the scanned volume (MPEG 4.9 MB). [URL: http://dx.doi.org/10.1117/1.3470241.6].

either the islet cellular structure or the microvasculature. Thus, additional imaging approaches, such as the 3-D optical method developed in this study, will need to fill the gap between the routine planar microscopy and the noninvasive imaging methods to provide insights into tissue's microstructure and networks.

\section{Conclusions}

In this report, we present an optical method for imaging the islet microstructure and vasculature in the pancreas. The integration of fluorescent labeling, optical clearing, confocal microscopy, and 3-D image rendering provides a unique approach to visualize the islet and its vascular network in situ. Unlike the microtome-based tissue imaging, this optical method for penetrative imaging of the pancreas yields clear, continuous optical sections for an integrated visualization of the islet microstructure and vascular network with subcellularlevel resolution. This new optical approach will change our conventional planar view of the islet structure into a 3-D panorama for better understanding the islet physiology and diabetes mellitus.

\section{Acknowledgments}

This work was supported in part by grants from the National Science Council (NSC 96-3011-P-007-006, 98-3011-P-007004, and 98-2221-E-007-035) and the 5-Year Research Program in NTHU, Taiwan.

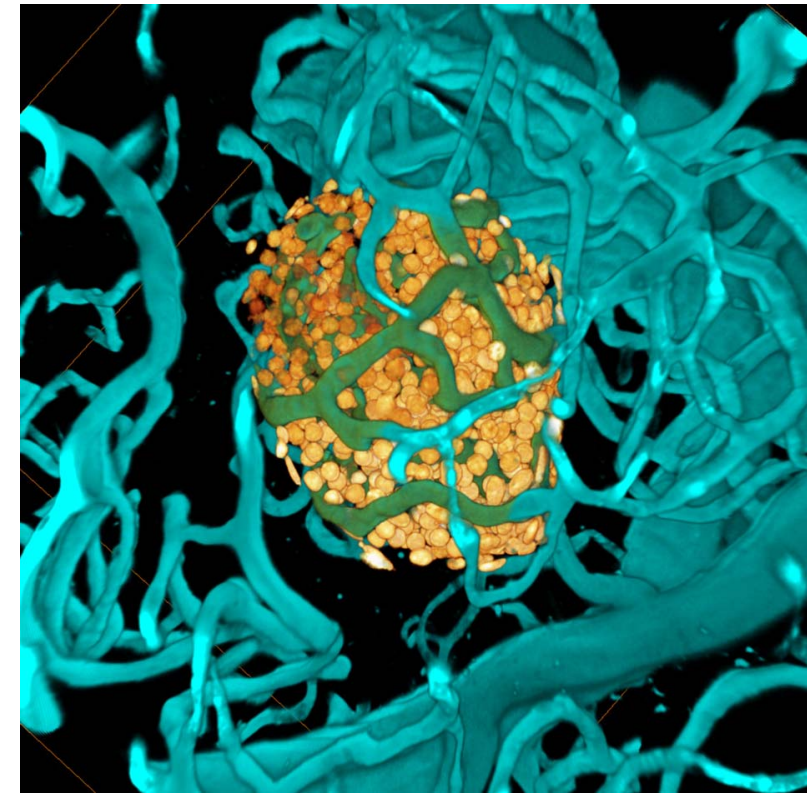

Video 7. Fly-through presentation of the vasculature in the interior and exterior domains of an islet. The interior domain of the islet vasculature was assigned to yellow, using the Image Segmentation function of the Amira software and the ovoid islet surface as the segmentation boundary. Dimensions of the scanned volume: $369 \mu \mathrm{m}(x)$ $\times 369 \mu \mathrm{m}(y) \times 207 \mu \mathrm{m}$ ( $z$, depth) (MPEG $5 \mathrm{MB})$. (Color online only.) [URL: http://dx.doi.org/10.1117/1.3470241.7].

\section{References}

1. O. Cabrera, D. M. Berman, N. S. Kenyon, C. Ricordi, P. O. Berggrern, and A. Caicedo, "The unique cytoarchitecture of human pancreatic islets has implications for islet cell function," Proc. Natl. Acad. Sci. U.S.A. 103(7), 2334-2339 (2006).

2. J. Gromada, I. Franklin, and C. B. Wollheim, "Alpha-cells of the endocrine pancreas: 35 years of research but the enigma remains," Endocr. Rev. 28(1), 84-116 (2007).

3. F. C. Brunicardi, J. Stagner, S. Bonner-Weir, H. Wayland, R. Kleinman, E. Livingston, P. Guth, M. Menger, R. McCuskey, M. Intaglietta, A. Charles, S. Ashley, A. Cheung, E. Ipp, S. Gilman, T. Howard, and E. Passaro, "Microcirculation of the islets of Langerhans-Long Beach Veterans Administration Regional Medical Education Center Symposium," Diabetes 45(4), 385-392 (1996).

4. N. Ballian and F. C. Brunicardi, "Islet vasculature as a regulator of endocrine pancreas function," World J. Surg. 31(4), 705-714 (2007).

5. V. V. Tuchin, "A clear vision for laser diagnostics," IEEE J. Sel. Top. Quantum Electron. 13(6), 1621-1628 (2007).

6. R. K. K. Wang, X. Q. Xu, V. V. Tuchin, and J. B. Elder, "Concurrent enhancement of imaging depth and contrast for optical coherence tomography by hyperosmotic agents," J. Opt. Soc. Am. B 18(7), 948953 (2001).

7. J. Y. Jiang, M. Boese, P. Turner, and R. K. K. Wang, "Penetration kinetics of dimethyl sulphoxide and glycerol in dynamic optical clearing of porcine skin tissue in vitro studied by Fourier transform infrared spectroscopic imaging," J. Biomed. Opt. 13(2), 021105 (2008).

8. E. A. Genina, A. N. Bashkatov, A. A. Korobko, E. A. Zubkova, V. V. Tuchin, I. Yaroslavsky, and G. B. Altshuler, "Optical clearing of human skin: comparative study of permeability and dehydration of intact and photothermally perforated skin," J. Biomed. Opt. 13(2), 021102 (2008).

9. M. G. Ghosn, E. F. Carbajal, N. A. Befrui, V. V. Tuchin, and K. V. Larin, "Differential permeability rate and percent clearing of glucose in different regions in rabbit sclera," J. Biomed. Opt. 13(2), 021110 (2008).

10. A. T. Yeh and J. Hirshburg, "Molecular interactions of exogeneous chemical agents with collagen-implications for tissue optical clearing," J. Biomed. Opt. 11(1), 014003 (2006). 
11. A. K. Bui, R. A. McClure, J. Chang, C. Stoianovici, J. Hirshburg, A. T. Yeh, and B. Choi, "Revisiting optical clearing with dimethyl sulfoxide (DMSO)," Lasers Surg. Med. 41(2), 142-148 (2009).

12. Y. C. Liu and A. S. Chiang, "High-resolution confocal imaging and three-dimensional rendering," Methods 30(1), 86-93 (2003).

13. H. H. Lin, J. S. Lai, A. L. Chin, Y. C. Chen, and A. S. Chiang, "A map of olfactory representation in the Drosophila mushroom body," Cell 128(6), 1205-1217 (2007).

14. Y. Wang, A. Mamiya, A. S. Chiang, and Y. Zhong, "Imaging of an early memory trace in the Drosophila mushroom body," J. Neurosci. 28(17), 4368-4376 (2008).

15. Y. Y. Fu, C. W. Lin, G. Enikolopov, E. Sibley, A. S. Chiang, and S. C. Tang, "Microtome-free 3-dimensional confocal imaging method for visualization of mouse intestine with subcellular-level resolution," Gastroenterology 137(2), 453-465 (2009).

16. S. J. Tseng, Y. H. Lee, Z. H. Chen, H. H. Lin, C. Y. Lin, and S. C. Tang, "Integration of optical clearing and optical sectioning microscopy for three-dimensional imaging of natural biomaterial scaffolds in thin sections," J. Biomed. Opt. 14(4), 044004 (2009).

17. Carl Zeiss Microimaging GmbH, "Visualizing the architecture of cells and tissues," Brochures for Laser Scanning Microscopy, available at http://www.zeiss.de/micro (2009).

18. D. J. Ravnic, X. Jiang, T. Wolloscheck, J. P. Pratt, H. Huss, S. J. Mentzer, and M. A. Konerding, "Vessel painting of the microcirculation using fluorescent lipophilic tracers," Microvasc. Res. 70(1-2), 90-96 (2005).

19. Y. Li, Y. Song, L. Zhao, G. Gaidosh, A. M. Laties, and R. Wen, "Direct labeling and visualization of blood vessels with lipophilic carbocyanine dye DiI," Nat. Protoc. 3(11), 1703-1708 (2008).

20. M. Hara, R. F. Dizon, B. S. Glick, C. S. Lee, K. H. Kaestner, D. W. Piston, and V. P. Bindokas, "Imaging pancreatic beta-cells in the intact pancreas," Am. J. Physiol._Endocrinol. Metab. 290(5), E1041E1047 (2006).

21. T. Alanentalo, C. E. Loren, A. Larefalk, J. Sharpe, D. Holmberg, and U. Ahlgren, "High-resolution three-dimensional imaging of isletinfiltrate interactions based on optical projection tomography assessments of the intact adult mouse pancreas," J. Biomed. Opt. 13(5), 054070 (2008).

22. J. L. Mignone, V. Kukekov, A. S. Chiang, D. Steindler, and G. Enikolopov, "Neural stem and progenitor cells in nestin-GFP transgenic mice," J. Comp. Neurol. 469(3), 311-324 (2004).

23. A. Delacour, V. Nepote, A. Trumpp, and P. L. Herrera, "Nestin expression in pancreatic exocrine cell lineages," Mech. Dev. 121(1), 3-14 (2004).
24. A. S. Chiang, "Aqueous tissue clearing solution," U.S. Patent No. 6,472,216 (2002).

25. N. S. Goldstein, A. Soman, and J. Sacksner, "Disparate surgical margin lengths of colorectal resection specimens between in vivo and in vitro measurements. The effects of surgical resection and formalin fixation on organ shrinkage," Am. J. Clin. Pathol. 111(3), 349-351 (1999).

26. D. Holmberg and U. Ahlgren, "Imaging the pancreas: from ex vivo to non-invasive technology," Diabetologia 51(12), 2148-2154 (2008).

27. J. Virostko and A. C. Powers, "Molecular imaging of the pancreas in small animal models," Gastroenterology 136(2), 407-409 (2009).

28. L. R. Nyman, K. S. Wells, W. S. Head, M. McCaughey, E. Ford, M. Brissova, D. W. Piston, and A. C. Powers, "Real-time, multidimensional in vivo imaging used to investigate blood flow in mouse pancreatic islets," J. Clin. Invest. 118(11), 3790-3797 (2008).

29. S. Speier, D. Nyqvist, O. Cabrera, J. Yu, R. D. Molano, A. Pileggi, T. Moede, M. Kohler, J. Wilbertz, B. Leibiger, C. Ricordi, I. B. Leibiger, A. Caicedo, and P. O. Berggren, "Noninvasive in vivo imaging of pancreatic islet cell biology," Nat. Med. 14(5), 574-578 (2008).

30. M. C. Denis, U. Mahmood, C. Benoist, D. Mathis, and R. Weissleder, "Imaging inflammation of the pancreatic islets in type 1 diabetes," Proc. Natl. Acad. Sci. U.S.A. 101(34), 12634-12639 (2004).

31. S. E. Turvey, E. Swart, M. C. Denis, U. Mahmood, C. Benoist, R. Weissleder, and D. Mathis, "Noninvasive imaging of pancreatic inflammation and its reversal in type 1 diabetes," J. Clin. Invest. 115(9), 2454-2461 (2005).

32. Z. Medarova, G. Castillo, G. Dai, E. Bolotin, A. Bogdanov, and A. Moore, "Noninvasive magnetic resonance imaging of microvascular changes in type 1 diabetes," Diabetes 56(11), 2677-2682 (2007).

33. F. Souza, M. Freeby, K. Hultman, N. Simpson, A. Herron, P. Witkowsky, E. Liu, A. Maffei, and P. E. Harris, "Current progress in non-invasive imaging of beta cell mass of the endocrine pancreas," Curr. Med. Chem. 13(23), 2761-2773 (2006).

34. M. M. Martinic and M. G. von Herrath, "Real-time imaging of the pancreas during development of diabetes," Immunol. Rev. 221, 200213 (2008).

35. M. Villiger, J. Goulley, M. Friedrich, A. Grapin-Botton, P. Meda, T. Lasser, and R. A. Leitgeb, "In vivo imaging of murine endocrine islets of Langerhans with extended-focus optical coherence microscopy," Diabetologia 52(8), 1599-1607 (2009).

36. W. J. Malaisse, K. Louchami, and A. Sener, "Noninvasive imaging of pancreatic beta cells," Nat. Rev. Endocrinol. 5(7), 394-400 (2009). 